\title{
Dielectric Relaxation Mechanism for Proton Glass
}

\section{Author: V. Hugo Schmidt}

This is an Accepted Manuscript of an article published in Ferroelectrics on February 1, 1988, available online: http://www.tandfonline.com/10.1080/00150198808215907.

Schmidt, V. Hugo. "Dielectric Relaxation Mechanism for Proton Glass." Ferroelectrics 78, no. 1 (February 1988): 207-214. doi: 10.1080/00150198808215907.

Made available through Montana State University's $\underline{\text { ScholarWorks }}$ scholarworks. montana.edu 
DIELECTRIC RELAXATION MECHANISM FOR PROTON GLASS

\title{
V. HUGO SCHMIDT
}

Physics Dept.. Montana State University, Bozeman, MT, U.S.A.

\begin{abstract}
Dielectric relaxation of proton glasses is controlled by $0-H . . .0$ proton intrabond hopping responsible for 'Takagi"' $\mathrm{HPO}_{4}$ and $\mathrm{H}_{3} \mathrm{PO}_{4}$ intrinsic defect diffusion. The diffusion path has a one-dimensional fractal topology. The defects diffuse in a potential which also has a fractal nature, giving a barrier height distribution leading to a wide spread in dielectric relaxation times at 10 w temperature. Expressions for dielectric relaxation and ac susceptibility are derived, and their fit to experimental results is brief $1 y$ discussed.
\end{abstract}

The proton $\mathrm{g} 1 \mathrm{ass}^{1} \mathrm{Rb}_{1-\mathrm{x}}\left(\mathrm{NH}_{4}\right) \mathrm{x}_{2} \mathrm{PO}_{4}$ (RADP) is a mixed crystal of $\mathrm{RbH}_{2} \mathrm{PO}_{4}$ (RDP) and $\mathrm{NH}_{4} \mathrm{H}_{2} \mathrm{PO}_{4}$ (ADP). Its constituents, $\mathrm{RDP}$ and ADP, have the same tetragonal paraelectric (PE) phase structure. They undergo ferroelectric (FE) and antiferroelectric (AFE) orderdisorder transitions at 146 and $148 \mathrm{~K}$ respectively which order the 'acid"' 0-H...o protons different1y. The mixed crystal RADP has no transitions for $0.22<x<0.74$, but proton glass (PG) behavior exhibiting a wide spread in dielectric and nuclear magnetic resonance (NMR) relaxation times sets in near $75 \mathrm{~K}$.

The dielectric behavior is described accurately by a phenomenological model ${ }^{1}$ with 7 adjustable parameters based on the Voge1-Fulcher 1aw. Presented here is a microscopic mode1 which explains some features of the dielectric relaxation. 
This mode1 has no adjustable parameters. It is based on three short-range interactions. The first is the Slater interaction which gives the nonpolar $\mathrm{H}_{2} \mathrm{PO}_{4}$ groups found in the AFE phase of ADP an energy $\varepsilon_{0}$ higher than the polar groups constituting the FE phase of RDP. It is assumed that $\varepsilon_{0} / k$ retains for all $x$ the value $74.5 \mathrm{~K}_{\text {reported }}{ }^{3}$ for pure RDP. The second (cross-cation) interaction $\varepsilon_{a}$ links two acid protons via an $\mathrm{NH}_{4}^{+}$(but not $\mathrm{Rb}^{+}$) cation. ${ }^{2}$ Its definition in Ref. 2 is correct. but its strength is $\varepsilon_{a}=\varepsilon_{0}$ and not $\varepsilon_{a}=2 \varepsilon_{a}$ as stated in Refs. 2 and 4. The third interaction is $\varepsilon_{1}$, half the creation energy for a Takagi $\mathrm{HPO}_{4}-\mathrm{H}_{3} \mathrm{PO}_{4}$ defect pair. ${ }^{4}$ Its value for a11 $x$ is assumed to be $\varepsilon_{1} / k=647 \mathrm{~K}$ as reported ${ }^{3}$ for pure RDP. All these proton-proton interactions can be represented by pseudospin interactions. 4

To summarize a review ${ }^{4}$ of effects of these interactions, the Slater interaction $\varepsilon_{0}$ alone gives the FE transition in RDP but cannot explain dynamic behavior. Adding the cross-cation $\varepsilon_{a}$ interaction predicts ${ }^{2}$ static behavior of RADP including the $x-T$ phase diagram if $\varepsilon_{0}$ is kept independent of $x$ and $\varepsilon_{a}$ is proportional to $x$ in that mean-field model. Explanation of dyamic behavior requires consideration also of Takagi pairs and their creation energy $2 \varepsilon_{1}$ in Monte Carlo simulations 5,6 and dielectric and NMR relaxation studies. 7.8

The chart below outlines the steps in the model calculation.

$$
\begin{gathered}
\left.\varepsilon_{d^{-\rightarrow U(r)}}^{\tau_{0}}{ }_{\left(D, \varepsilon_{1}\right)}\right\} \rightarrow\left(R_{0}, n\right) \\
j \rightarrow P(t)-\rightarrow\left(\varepsilon^{\prime}, \varepsilon^{\prime \prime}\right)
\end{gathered}
$$

Following the flow chart from left to right, $\varepsilon_{d}$ is the rms defect diffusion energy per diffusion step. Its mean square value is given by 


$$
\varepsilon_{\mathrm{d}} 2=\left\langle\varepsilon^{2}\right\rangle=2 \times \varepsilon_{\mathrm{a}} 2+2 \mathrm{f}_{\mathrm{g}}\left(1-\mathrm{f}_{\mathrm{g}}\right) \varepsilon_{0}{ }^{2}
$$

Here $x=0.35$ is the ammonium fraction in crystals used by Courtens et a1. in dielectric ${ }^{1}$ and Brillouin scattering ${ }^{9}$ studies whose results are compared with predictions of this model. The factor $f_{g}=0.77291$ is the limiting fraction ${ }^{2}$ of zero-energy slater groups in the PG regime. In pure ADP a proton transfer has probability $1 / 2$ each of changing $U$ by $\pm 2 \varepsilon_{a}$ and of leaving $U$ unchanged, giving the first term in Eq. (1). The second term assumes that the probability of creating or annibilating a slater group of energy 0 or $\varepsilon_{0}$ is proportional to the respective fractions $f_{g}$ and $1-f_{g}$ of those groups already present. Because $\varepsilon_{\mathrm{a}}=\varepsilon_{0}=74.5 \mathrm{~K}$ in our mode 1 as discussed above, we obtain the value $\varepsilon_{d}=76.4 \mathrm{~K}$.

The $\varepsilon$ distribution leading to Eq. (1) is discrete but nearly equivalent to the normalized gausian form

$$
w(\varepsilon)=(2 \pi)^{-1 / 2} \varepsilon_{d}-1 \exp \left[-\left(\varepsilon-\varepsilon_{b}\right)^{2} / 2 \varepsilon_{d}^{2}\right]
$$

The bias energy $\varepsilon_{b}$ in thermal equilibrium must be such that defect diffusion on the average does not change the internal energy $U$.

The distribution $W(\varepsilon)$ of internal energy change $\varepsilon$ per step along defect diffusion paths actually taken is found by assuning

$$
W(\varepsilon)=w(\varepsilon) \exp (-\varepsilon / k T)
$$

The assumption that $U$ is unbiased along paths actually taken then determines the value of $\varepsilon_{b}$ and gives $W(\varepsilon)$ the form

$$
W(\varepsilon)=(2 \pi)^{-1 / 2} \varepsilon_{\mathrm{d}}{ }^{-1} \exp \left(-\varepsilon^{2} / 2 \varepsilon_{\mathrm{d}}{ }^{2}\right) \text { if } \varepsilon_{\mathrm{b}}=\varepsilon_{\mathrm{d}}^{2} / \mathrm{kT}
$$

If motion of other Takagi defects is neglected, we can equate $U$ to an internal energy potential $U(r)$ of a defect $r$ steps along its diffusion path away from its original site. Although J(r) 
defined in this way is single-valued, $U$ for a given defect at a given site can be maltivalued because the change in $U$ in going to a new site depends on the path. The 2-d analog of this potential is a depression with caves in the surrounding slopes, so that though an outwara path ( $A$ in Fig. 1) chosen randomly using $w(\varepsilon)$ is uphill, a typical actual outward path (B in Fig. 1) using W( $\varepsilon$ ) is level on the average.

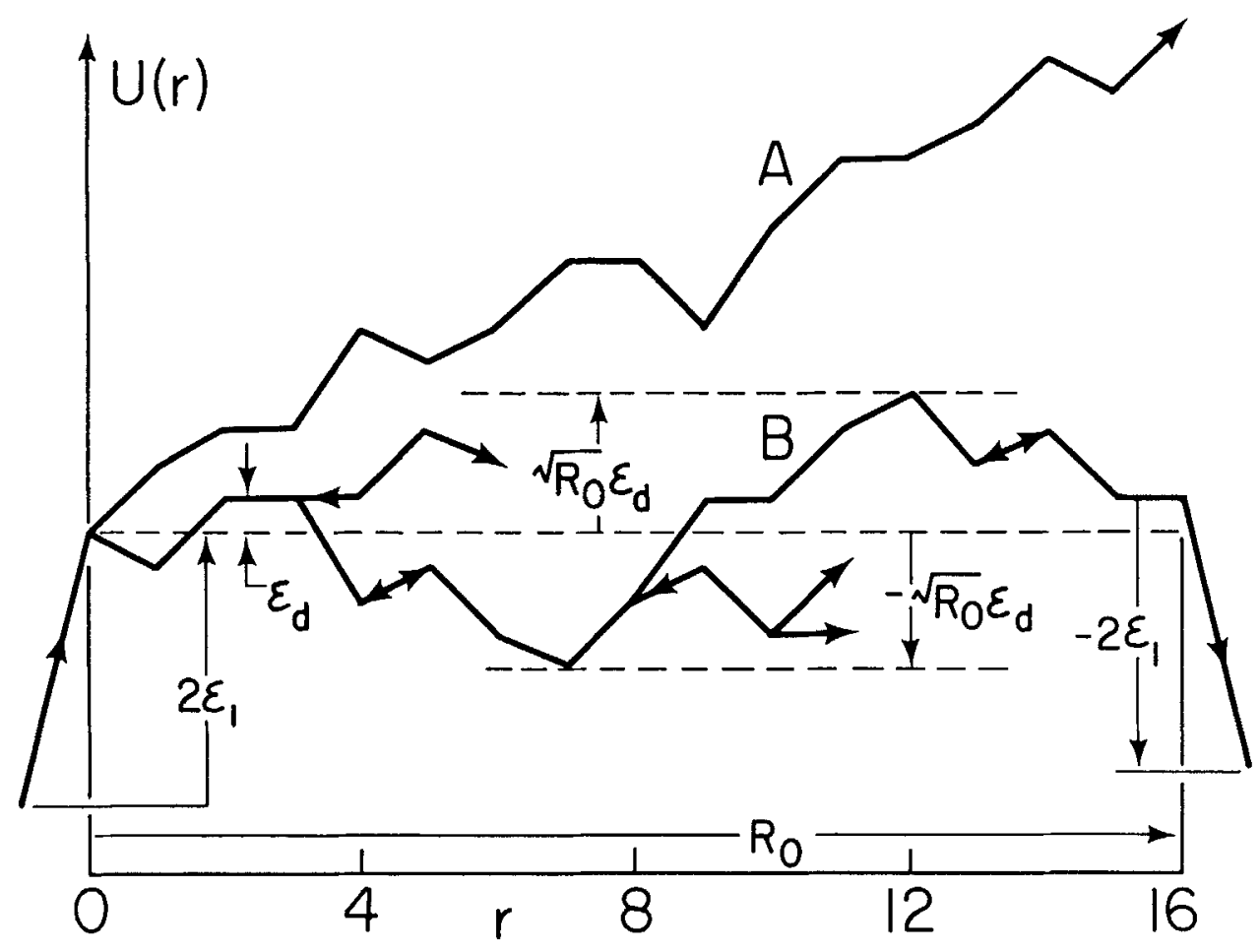

FIGURE 1 Typical Takagi defect diffusion path showing creation, diffusion and annihilation and illustrating parameters discussed in text.

The diffusion paths available to a defect locally have the topology of a double-branching Cayley tree (Bethe 1attice) because 
a defect can move to two new positions or return to its former site. We made a Monte Carlo study of diffusion on such a Cayley tree for a number of paths of maximum length $r=13$ steps from the origin. These simulations indicate that the number $\mathrm{N}_{s}$ of new sites visited obeys the fractal relation $N_{s}=r^{D}$, with Hausdorff dimensionality ${ }^{10} D=1.15 \pm 0.05$. A value 1 for $D$ would correspond to one-dimensional (1-d) diffusion with no side trips.

A defect can take a branch path as shown in Fig. 1, but retracing the path cancels the polarization changes made while taking that branch. Accordingly the net path causing polarization change is at any instant strictly 1-d. Because $U(r)$ along such 1-d paths obeys the unbiased $z$ distribution of Eq. (4), U(r) is a fractal potential of a type considered by Dotsenko. ${ }^{11}$

The defect diffusion rate depends on the Boltzmann factors found from $U(r)$ for the defect's three possible new sites, and on the attempt time $\tau_{0}$. Brillouin scattering studies by Courtens et a1. 9 fix $\tau_{0}$ at $5 \times 10^{-14}$ s.

Because defect diffusion has the net effect for purposes of dielectric relaxation of reversing the dipoles along a 1-d path, we define a diffusion path length $R(t)$ of number of steps taken in time interval t. The maximum barrier encountered in $R$ steps, a parameter important for dielectric relaxation, is about $\varepsilon_{d} R^{1 / 2}$ as shown in Fig. 1 for the W(E) distribution of $\mathrm{Bq}$. (4).

These barriers are negligible at high temperature, so the defect $j$ ump time is simply $\tau_{0}$ and it requires a time near $\tau_{0} R^{2}$ to diffuse $R$ steps, thus giving the usual diffusion relation. But in the $P G$ regime the largest barrier dominates the diffusion time, so the factor $R^{2}$ should be omitted, giving diffusion time $t(R)$ and diffusion rate $R(t)$ shown below:

$$
t(R)=\tau_{0} \exp \left(\varepsilon_{d} R^{1 / 2} / k T\right), R(t)=\left\langle R^{2}\right\rangle^{1 / 2}=\left[\left(k T / \varepsilon_{d}\right) \ln \left(t / \tau_{0}\right)\right]^{2}
$$

These equations are strictly valid only for $1-d$ systems, ${ }^{11}$ but 
apply here because the defect diffusion path is almost 1-d so that the maximum barrier is unlikely to be in a branch path.

The fractional Takagi defect density $n$ is found from the Boltzmann factor for such groups, using the fact that $n<<1$ :

$$
n=\exp \left[-\left(\varepsilon_{1}-R_{0}^{1 / 2} \varepsilon_{d}\right) / k T\right]
$$

For the Takagi defect creation energy $\varepsilon_{1}$ we choose $647 \mathrm{~K}$ as discussed above. The average defect annihilation path length $R_{0}$ shown in Fig. 1 is the path length in number of steps from creation to annihilation with new partner. This path length determines the size of the mean trapping energy $-R_{0}^{1 / 2} \varepsilon_{d}$ also shown in Fig. 1, by the same argument used above to find the maximum barrier height $+R^{1 / 2} \varepsilon_{d}$.

The second relation needed to solve for $R_{0}$ and $n$ comes from the above relation between $N_{s}$ and $r$. We set $r=R_{0}$ and find $N_{s}$ by noting that the defect has probability near $n$ of annibilating with a new partner at each new site visited, so on the average it will visit $N_{s}=n^{-1}$ sites, giving the relation

$$
\mathrm{n}^{-1}=\mathrm{R}_{0}{ }^{\mathrm{D}}
$$

Both $n$ and $R_{0}$ are found from Eqs. (6) and (7), and $n$ is

$$
n=\left\{\left[\varepsilon_{1}+k T 1 n(n)\right] / \varepsilon_{d}\right\}^{-2 D}
$$

The polarization relaxation $P(t)$ following step removal at $t=0$ of a small dc electric field is found by integrating the fractional polarization change $d P / P$ which equals ndR(dt) if the defect wanders randomly, as at high temperature. Here dR(dt) is the mean number of dipoles reversed by a defect during time dt.

Near the FE transition temperatare $T_{c}$ for Rb-rich crystals the defect path is nonrandom and because RDP-type crystals 
generally exhibit mean-field behavior, the relaxation is expected ${ }^{12}$ to show Curie-Weiss behavior:

$$
\mathrm{dP} / \mathrm{P}=-\left[\left(\mathrm{T}-\mathbf{T}_{\mathbf{c}}\right) / \mathrm{T}\right] \mathbf{n d R} \text {. }
$$

Such slowing down of polarization decay near $T_{c}$ was seen in Monte Carlo simulations on the Rb-rich side of the phase diagram. ${ }^{6}$

For mixed crystals in the $P G$ concentration range, $T_{c}$ can be approximated by 0 . Integration of $\mathrm{Eq}$. (9) then yields $1 n(P)+$ const $=-n R$. Substitution of $R(t)$ from $E q$. (5) and taking the exponent provides the following expression for polarization decay from an initial value $P_{i}$ :

$$
P / P_{i}=\exp \left\{-n\left[\left(k T / \varepsilon_{d}\right) \ln \left(1+t / \tau_{0}\right)\right]^{2}\right\}
$$

This decay has $1 n^{2}(t)$ in the exponent. A form with arbitrary power of $1 \mathrm{n}(t)$ was derived by Dotsenko, 11 and by Palmer et a1. using two models employing hierarchically constrained dynamics 13 and relaxation of isolated clusters of unfrustrated spins. 14

The ac response is found from $\mathrm{Eq}$. (10) by integrating the responses at time to an ac electric field of angular frequency $w$ made up of differential steps beginning at times $t$ '< $t$. The complex dielectric susceptibility $\varepsilon=\varepsilon^{\prime}-j \varepsilon^{\prime}$ ' found in this way is

$$
\varepsilon=\varepsilon_{\infty}+\left(\varepsilon_{d c}-\varepsilon_{\infty}\right)\left\{1-\int_{0}^{\infty}(\operatorname{sinu}+j \cos u) \exp \left[-a^{2} 1 n^{2}(1+u / f)\right] d u\right\}
$$

where $n=\omega\left(t-t^{\prime}\right), a=k T n^{4 / 2} / \varepsilon_{d}, f=\omega \tau_{0}$, and $\varepsilon_{d c}$ and $\varepsilon_{\infty}$ are the $\varepsilon^{\prime}$ values at temperatures just above and below the dispersion region.

We compared predictions of this model with andio frequency dielectric results ${ }^{1}$ and with the relaxation time range found 9 by a Brillouin scattering stady at $\mathrm{GHz}$ frequencies. We used $\tau_{0}=5 \times 10^{-14}$ s, $D=1.15, \varepsilon_{\mathrm{d}} / \mathrm{k}=76.4 \mathrm{~K}$ and $\varepsilon_{1}=647 \mathrm{~K}$ as discussed above. The fit was qualitatively correct, but was improved considerably by 
reducing the latter two parameters to $50 \mathrm{~K}$ and $400 \mathrm{~K}$ respectively. A graphical comparison has been submitted for publication elsewhere. The overal1 fit is quite satisfying, because only two of the four model parameters had to be changed somewhat. Most important, this fit was made with a realistic microscopic model. G. F. Tuthill and $S$. Cameron kindly planned and carried out the Monte Carlo defect diffusion simulation. R. Blinc and S. Zumer are thanked for helpful discussions.

\section{References}

1. E. Courtens, Phys. Rev. Lett. 52, 69 (1984).

2. V. H. Schmidt, J. T. Wang, and P. T. Schnackenberg. Jpn. J. App1. Phys. 24. Supp1. 24-2, 944 (1985).

3. C. W. Faira11 and W. Reese, Phys. Rev. B 11, 2066 (1975).

4. V. H. Schmidt, Ferroelectrics 72,157 (1987).

5. W. Selke and E. Courtens, Ferroelectrics Ltrs. 5, 173 (1986).

6. V. H. Schmidt and P. T. Schnackenberg, unpublished work.

7. E. Matsushita and T. Matsubara, Prog. Theor. Phys. 71, 235 (1984).

8. J. Slak, R. Kind, R. Blinc, E. Courtens, and S. Žumer, Phys. Rev. B $\underline{30}, 85$ (1984).

9. E. Courtens, R. Vacher, and Y. Dagorn, Phys. Rev. B $\underline{3}, 7625$ (1986).

10. On Growth and Form, H. E. Stanley and N. Ostrowsky. Eds. (Nijhoff, Boston, 1986), pp. 293 and 301.

11. V. S. Dotsenko, J. Phys, C 18, 6023 (1985).

12. H. E. Stanley, Introdaction to Phase Transitions and Critical Phenomena (Oxford, New York, 1971), p. 285 .

13. R. G. Palmer, D. L. Stein, E. Abrahams, and P. W. Anderson. Phys. Rey. Lett. 53, 958 (1984).

14. M. Randeria, J. P. Sethna, and R. G. Palmer. Phys. Rev. Lett. 54, 1321 (1985). 